\title{
PYOCOLPOS IN INFANCY
}

BY

IAN P. TODD, M.D., M.R.C.S.

(Resident Surgical Officer, The Hospital for Sick Children, Great Ormond Street, London)

Pyocolpos is rare at any age; in infancy it is extremely uncommon, and it is therefore desirable that every case should be reported, since the treatment is very simple when once the condition has been recognized.

Pyocolpos may be defined as ' a congenital atresia of the vaginal orifice, with retention of an excess of secondarily-infected cervical secretions'.

Case report. D. S., born June 26, 1945, was admitted to hospital at the age of $7 \frac{1}{2}$ hours. It was noticed, at birth, that 'something came down' into the vulva when the baby cried; it was for this reason that the child was sent immediately to hospital. She had not passed urine or faeces.

ON EXAMINATION. The child appeared to be quite healthy; the only abnormality found was that at the vulva. There was no abdominal swelling. Local examination revealed a cystic mass, anterior to the fourchette; this was assumed to be protruding or prolapsing from the vagina. It obscured the urethra. The labia majora and minora were normal. The swelling became more prominent when the child cried. The house-surgeon observed that a probe could be passed up some distance anteriorly; this suggested the diagnosis of rectocoele. Digital examination of the rectum showed, however, that there was no prolapse of the anterior wall of the rectum. The most likely diagnosis, therefore, appeared to be that of cyst in the rectovaginal septum.

The infant soon passed urine and faeces normally, and was discharged to attend the Out-patient Department.

She was re-admitted on October 6,1945 , at the age of $3 \frac{1}{2}$ months, in poor condition, having passed no urine for twenty-four hours. Examination showed the same condition as before; but, in addition, there was a large tumour in the mid-line of the lower abdomen, almost reaching the level of the umbilicus. It was assumed that the tumour was the bladder, and the child was taken at once to the operating theatre and was examined under anaesthesia. The urethra was found with some difficulty, since it had been pulled upward. A silver catheter was passed. Concentrated urine flowed; but though the tumour became smaller it did not disappear, and a cystic mass remained, in the mid-line and arising from the pelvis. Fluctuation could not be elicited between this swelling and the vulval tumour.

Nothing more was done for the time being owing to the child's poor condition. Catheters were passed without difficulty three times in each twenty-four hours for several days.
The abdomen was opened on October 10, 1945 , through a left lower para-median incision. The bladder presented; it was aspirated. The wall was thickened, loculated and trabeculated. Just behind the bladder, and attached below by peritoneum, was a firm, cystic swelling in the mid-line; this was surmounted by a small, undistended uterus, with normal adnexae.

Cross-fluctuation was found to be present between this and the vulval swelling. The latter was then aspirated; pus was withdrawn. An incision was, therefore, made into it, and a Malecot catheter was inserted. Much very foul-smelling pus was evacuated. The abdominal tumour, which was obviously the distended vagina, collapsed. The laparotomy wound was then closed.

The child recovered well from the operation.

The pus contained a large number of grampositive cocci and $\mathrm{B}$. proteus vulgaris. The cavity was, therefore, washed out twice a day with saline solution, and Penicillin was instilled. The discharge ceased on October 31, and the catheter was removed. The vaginal orifice was dilated under anaesthesia; the vagina was found to be abnormally long, but the cervix was normal.

The infant was discharged on November 4, 1945, and has since then attended the Out-patient Department twice. She is now well and is gaining weight, and the vaginal orifice appears to be normal.

Discussion. The condition, in this case, seems to have been one of true pyocolpos, for the uterus was not involved in any way. This was not so in the four uninfected cases reported by Mahoney and Chamberlain (1940), though they had an opportunity of examining two of their specimens histologically. They found that the condition was due to atresia of the vaginal orifice, comparable with imperforate anus, and not to imperforate hymen. This would be more in keeping with embryological evidence.

It is interesting, in the present case, to postulate the mode of infection of the closed cavity. The infection was, in all probability, blood- or lymphborne from the lower bowel.

Very few cases of this type have been recorded. Commandeur (1904) quoted ten cases by various authors. Cranwell (1905), Bjerrum (1915), Spencer (1916), Bonnet (1930), and Mahoney and Chamberlain (1940) have reported cases since then; some of them were infected, though the majority were not.

A case similar to that now recorded has 
recently been treated at King's College Hospital. A cystogram, as suggested by Mahoney and Chamberlain, is a useful aid to diagnosis; it shows the gross forward displacement of the bladder. More important, however, is the recognition of the clinical entity; and then follows the treatment, which is both simple and effective.

Summary. A case of pyocolpos in infancy is recorded. The symptoms and signs of the condition, and its treatment, are given. Stress is laid upon the importance of recognition of the clinical condition, and upon the simplicity and adequacy of the treatment.

I should like to thank Mr. T. Twistington Higgins and Mr. Alan Small for their permission to publish this case.

\section{REFERENCES}

Bjerrum (1915). Ugesk. f. Laeger. Kbenh., 77, 407. Bonnet, A. (1930). Thesis, Paris.

Commandeur, M. (1904). Bull. de la Soc. d'Obstet de Paris, $7,54$.

Cranwell, D. J. (1905). Rev. Gynec. et de' Chir. abd., 9, 635.

King's College Hospital (1946). Personal communication.

Mahoney, P. I., and Chamberlain, J. W. (1940). J. Pediat., 17, 772.

Spencer, H. R. (1916). Lancet, 1, 823. 